\title{
STUDY OF MACROINVERTEBRATE FUNCTIONAL FEEDING GROUP ABUNDANCE IN TUANJIE RESERVOIR OF NORTHEAST CHINA
}

\author{
CHEN, Q. ${ }^{1,2}-$ SUN, $X .^{1}-$ YU, H. X..$^{1 *}$ \\ ${ }^{1}$ College of Wildlife and Protected Area, Northeast Forestry University, Harbin 150040, China \\ ${ }^{2}$ Heilongjiang Forestry and Grassland Bureau, Harbin 150090, China \\ ${ }^{*}$ Corresponding author \\ e-mail: china.yhx@163.com \\ (Received $12^{\text {th }}$ Dec 2019; accepted $6^{\text {th }}$ May 2020)
}

\begin{abstract}
In this study, the concept of functional feeding groups was used to classify macroinvertebrate community structure. A total of 55 genera or species were sampled in the Tuanjie Reservoir in China and identified into six functional feeding groups. The mean values of water transparency (SD), dissolved oxygen (DO), $\mathrm{pH}$, water temperature (T), total phosphorus (TP), chemical oxygen demand $\left(\mathrm{COD}_{\mathrm{Mn}}\right)$, dissolved copper $\left(\mathrm{Cu}^{2+}\right)$, depth (D), electrical conductivity (EC), total nitrogen (TN) and dissolved iron $\left(\mathrm{Fe}^{3+}\right)$ showed significant difference between sampling sites $(P<0.05)$, while ammonium nitrogen $\left(\mathrm{NH}_{4}{ }^{+}-\mathrm{N}\right)$ and nitrate nitrogen $\left(\mathrm{NO}_{3}{ }^{-}-\mathrm{N}\right)$ were not significantly different $(P>0.05)$. Macroinvertebrate samples were also different, we find that the highest abundance was observed in summer at all sampling sites except S5 which was documented in spring, while the lowest abundance recorded in autumn except for S1 (in spring). Pearson and redundancy analysis (RDA) results showed that $\mathrm{T}, \mathrm{NH}_{4}^{+}-\mathrm{N}, \mathrm{Fe}^{3+}, \mathrm{DO}$ and $\mathrm{COD}_{\mathrm{Mn}}$ were the major factors influencing zooplankton functional groups in Tuanjie Reservoir.
\end{abstract}

Keywords: macroinvertebrate, functional diversity, environmental factors, drinking water, correlation

\section{Introduction}

Macroinvertebrates play an important role in the aquatic ecosystem, which is an important channel for nutrient recycling and energy flow to higher levels (Benke et al., 2010). Aquatic ecosystems are mostly affected by agricultural non-point source pollution which poses severe threats to macroinvertebrates (Shabani et al., 2019). The diversity of macroinvertebrates community structure can reflect the disturbance degree of long-term human activities aquatic ecosystem (Plafkin et al., 1989). Macroinvertebrates are widely used to monitor the damage of aquatic ecosystem. They are also an important part of aquatic food web and the basis of nutrient cycle and ecological balance of ecosystem (Liu et al., 2012; Mangadze et al., 2016; Hu et al., 2018). Benthic animals are the food source of aquatic animals such as fish and play an important role in fishery production. Therefore, the investigation of benthic animals in reservoirs has important reference value for understanding the nutritional status and water productivity of reservoirs. Benthic animals are the food source of aquatic animals such as fish and play an important role in fishery production. Therefore, the investigation of benthic animals in reservoirs has important reference value for understanding the nutritional status and water productivity of reservoirs (Chi et al., 2009).

Functional groups are classified based on physiological, morphological, life history or other biological characteristics associated with an ecosystem and with the behavior of species, and are aggregates of all species with similar functions in the community (An et 
al., 2016). Because of the environmental and spatial scale changes caused by nature and human beings, aquatic organisms respond accordingly. Researchers classify the priority functional groups of species according to similar biological and ecological characteristics, and these characteristics are consistent with the gradient of the environment (Poff et al., 2006). Species characteristics of functional groups are more closely related to the environment, which can more directly reflect the ecological process of ecological environment affecting aquatic communities, and can understand the aquatic ecosystem and its biodiversity (An et al., 2017).

China is the country with the largest number of reservoirs in the world (Liu et al., 2012). In many provinces, reservoirs have become an important source of water supply (Han, 2010), and are considered as the last barrier for drinking water safety in China and even for human beings (Han et al., 2016). Although there are a large number of reservoirs in China, the importance of reservoirs is increasing day by day, but relative to lakes and rivers, the research on benthic zoology of reservoirs is still scarce and needs to be strengthened (Liu et al., 2012).

Tuanjie Reservoir was built in 1981 with the aim of supplying drinking water to the local village people. The main sources water of the reservoir is Muling River, which is the largest tributary of the Ussuri River on the left bank of the border between China and Russia. Recently, the ecological study of Tuanjie reservoir mainly focuses on plankton (Chen et al., 2019; Sun et al., 2019). In this study, the macroinvertebrate functional feeding groups (FFGs) were used to reveal the relationship between macroinvertebrate FFGs abundance and environmental factors in Tuanjie Reservoir. We aim to collect macroinvertebrate fauna, and explore the relationships between macroinvertebrate functional feeding groups and environmental factors in the Tuanjie Reservoir.

\section{Materials and methods}

\section{Study area}

Tuanjie Reservoir $\left(130^{\circ} 8^{\prime}-130^{\circ} 11^{\prime} \mathrm{E}, 44^{\circ} 01^{\prime}-44^{\circ} 04^{\prime} \mathrm{N}\right)$ is located in the southeast of Heilongjiang Province Northeastern China (Fig. 1). The reservoir was built in 1981 in order to control floods, provide water for irrigation, fish farming, power generation and for aesthetic value. Tuanjie Reservoir has a surface area of $445 \mathrm{~km}^{2}$, a capacity of $8.63 \times 10^{7} \mathrm{~m}^{3}$ and it shaped like big "Y". The region where the reservoir is located is influenced by temperate continental monsoon. The average annual evaporation and precipitation of the reservoir are $950 \mathrm{~mm}$ and $534 \mathrm{~mm}$, respectively. The annual mean temperature is $1^{\circ} \mathrm{C}$, which ranged between $-44.1^{\circ} \mathrm{C}$ to $37.6^{\circ} \mathrm{C}$. In winter, the surface water of the reservoir is covered by ice (Sun et al., 2019).

\section{Environmental factors data sampling}

We collected all samples three times from 5 sampling sites of Tuanjie Reservoir on $6^{\text {th }} \sim 13^{\text {th }}$ May, $14^{\text {th }} \sim 21^{\text {th }}$ July and $20^{\text {th }} \sim 27^{\text {th }}$ September periods for spring, summer and autumn three seasons in 2015 (Table 1; Fig. 1). At each sampling site, water temperature (T), $\mathrm{pH}$, electrical conductivity (EC), dissolved oxygen (DO) was measured in the field using a portable multi-probe (YSI 6600, YSI Inc.). Water transparency (SD) and depth (D) were measured using Secchi disk and longline method. Triplicate water samples for chemical analyses were collected at each sampling sites and put on 
acid-washed plastic bottles, placed in ice box and transported to laboratory for analysis. The concentration of total nitrogen (TN), total phosphorus (TP), ammonium nitrogen $\left(\mathrm{NH}_{4}{ }^{+}-\mathrm{N}\right)$, nitrate nitrogen $\left(\mathrm{NO}_{3}{ }^{-}-\mathrm{N}\right)$, chemical oxygen demand $\left(\mathrm{COD}_{\mathrm{Mn}}\right)$ and dissolved iron $\left(\mathrm{Fe}^{3+}\right)$ and dissolved copper $\left(\mathrm{Cu}^{2+}\right)$ were measured according to the standard methods for China (MEP, 2002).

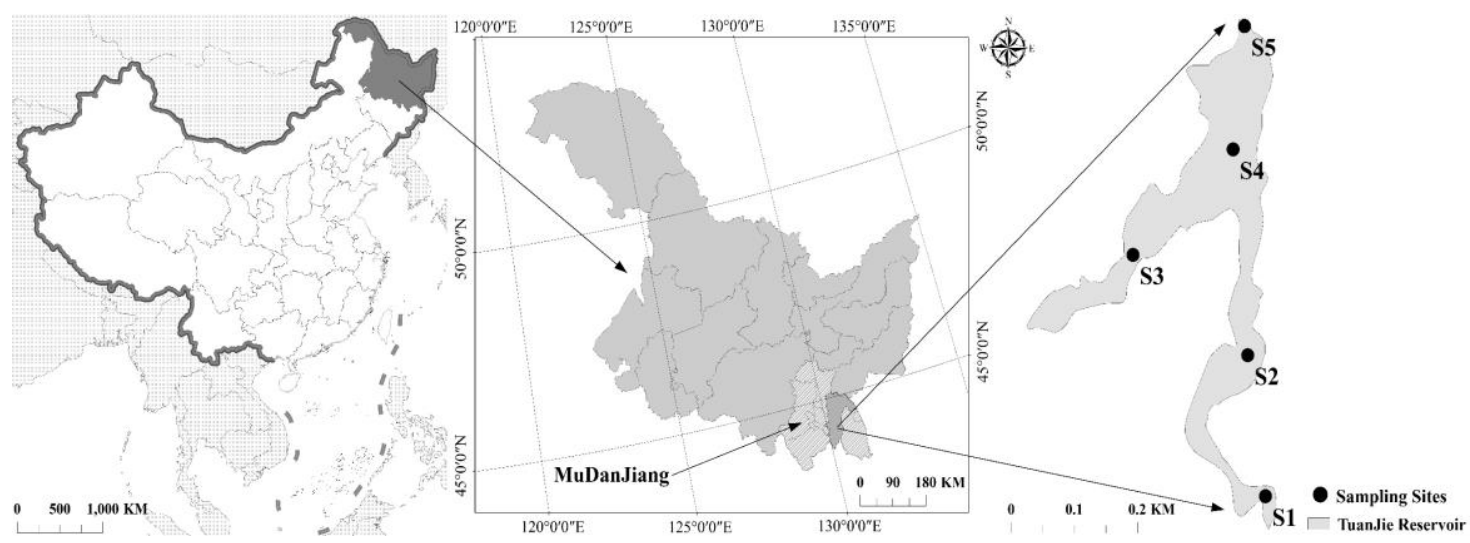

Figure 1. Map of sampling sites in Tuanjie Reservoir

Table 1. Five sampling sites coordinate in Tuanjie Reservoir in May (spring), July (summer) and September (autumn) in 2015

\begin{tabular}{|c|c|c|}
\hline Sampling sites & Latitude & Longitude \\
\hline $\mathrm{S} 1$ & $\mathrm{~N} 44^{\circ} 01^{\prime} 48^{\prime \prime}$ & E130 $11^{\circ} 24^{\prime \prime}$ \\
\hline S2 & 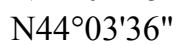 & $\mathrm{E} 130^{\circ} 10^{\prime} 48^{\prime \prime}$ \\
\hline S3 & $\mathrm{N}^{\prime} 4^{\circ} 03^{\prime} 00^{\prime \prime}$ & E13009'36" \\
\hline S4 & N4404'12" & $\mathrm{E} 130^{\circ} 10^{\prime} 36^{\prime \prime}$ \\
\hline S5 & N44ㅇํ' $04^{\prime} 48^{\prime \prime}$ & $\mathrm{E} 130^{\circ} 10^{\prime} 48^{\prime \prime}$ \\
\hline
\end{tabular}

\section{Macroinvertebrate FFGs data sampling}

Three random subsamples were collected in comparable habitat at locations of $1 \mathrm{~m}^{2}$ at each sampling site by using a Peterson mud bottom sampler $\left(\mathrm{CN}-150,1 / 16 \mathrm{~m}^{2}\right)$. All macroinvertebrates samples were composited into a single sample, preserved in $75 \%$ ethanol and transported to the laboratory for identification. In the laboratory, all samples were sorted on white porcelain pans, identified, and counted with a light stereomicroscope. All individuals were identified to genera or species using appropriate identification guides (Morse et al., 1994; Epler, 2001). Taxa were divided into six functional feeding groups (FFGs) according to Cummins et al. (1974) and Duan et al. (2010): predators (PR), omnivores (OM), gatherers/collectors (GC), filterers/collectors (FC), scrapers (SC) and shredders ( $\mathrm{SH})$.

\section{Data analysis}

Statistical analyses were carried out using the SPSS 19.0 software. Variation and correlation of environmental factors and macroinvertebrate FFGs abundance in different sampling sites were analyzed by using One-way ANOVA. Relationship between macroinvertebrate FFGs abundance and environmental factors was done using CANOCO 4.5 software (Microcomputer Power, New York, USA). Before analysis, the 
biological and abiotic data were transformed by $\log _{10}(x+1)$ to satisfy the normal distribution. We found that the detrended corresponding analysis (DCA) of the largest gradient length of the four axes was $0.648(<3)$. Therefore, linear ordination method of the redundancy analysis (RDA) was used to reveal the relationship. Monte Carlo simulations with 499 permutations were used to test the significance of the environmental factors in explaining the macroinvertebrate FFGs abundance in the RDA.

\section{Results and discussion}

\section{Environmental factors data characteristics}

The results of environmental factors mean values were shown in Table 2 and Table 3. Many biotic and abiotic factors, such as sediment type, water depth, food, dissolved oxygen, etc., have an important impact on the distribution of benthos community in lakes and reservoirs (Ji et al., 2015). There are many factors affecting the community structure, density, biomass and biodiversity of macroinvertebrates, special some important physical and chemical factors change (Petridis and Sinis, 1993; Buss et al., 2002; Duran, 2006). The cold climate in the North determines that the impact of temperature on macroinvertebrates is higher than that in other areas (Wang et al., 2019). The mean values of $\mathrm{SD}, \mathrm{DO}, \mathrm{pH}, \mathrm{T}, \mathrm{TP}, \mathrm{COD}_{\mathrm{Mn}}$ and $\mathrm{Cu}^{2+}$ were extremely significant difference among sampling sites $(P<0.01)$. And the mean values of $\mathrm{D}, \mathrm{EC}, \mathrm{TN}$ and $\mathrm{Fe}^{3+}$ showed significantly difference among sampling sites $(P<0.05)$. While $\mathrm{NH}_{4}{ }^{+}-\mathrm{N}$ and $\mathrm{NO}_{3}{ }^{-}-\mathrm{N}$ were not difference among sampling sites $(P>0.05)$ (Table 2). Among sampling seasons, the mean values of $\mathrm{D}, \mathrm{EC}, \mathrm{TN}, \mathrm{NO}_{3}^{-}-\mathrm{N}$ were not difference $(P>0.05)$, and $\mathrm{Cu}^{2+}$ showed significantly difference $(P<0.05)$. The other environmental factors were all extremely significant difference $(P<0.01)$ (Table 3$)$.

Table 2. The values (mean \pm SE) of environmental factors among sampling sites in May (spring), July (summer) and September (autumn) in 2015

\begin{tabular}{c|c|c|c|c|c|c|c}
\hline & S1 & S2 & S3 & S4 & S5 & $\boldsymbol{F}$ & $\boldsymbol{p}$ \\
\hline $\mathrm{SD}(\mathrm{m})$ & $0.66 \pm 0.10$ & $0.85 \pm 0.05$ & $0.95 \pm 0.06$ & $1.36 \pm 0.04$ & $1.46 \pm 0.05$ & 9.364 & $0.000^{* *}$ \\
$\mathrm{D}(\mathrm{m})$ & $0.84 \pm 0.07$ & $7.42 \pm 0.08$ & $2.44 \pm 0.07$ & $17.48 \pm 0.08$ & $22.72 \pm 0.07$ & 2.895 & $0.034^{*}$ \\
$\mathrm{EC}(\mathrm{mS} / \mathrm{cm})$ & $0.31 \pm 0.03$ & $0.08 \pm 0.01$ & $0.15 \pm 0.01$ & $0.10 \pm 0.01$ & $0.08 \pm 0.01$ & 2.633 & $0.048^{*}$ \\
$\mathrm{DO}(\mathrm{mg} / \mathrm{L})$ & $9.10 \pm 0.21$ & $8.27 \pm 0.09$ & $8.90 \pm 0.21$ & $9.00 \pm 0.26$ & $8.33 \pm 0.07$ & 5.016 & $0.002^{* *}$ \\
$\mathrm{pH}$ & $7.69 \pm 0.09$ & $7.65 \pm 0.04$ & $7.71 \pm 0.06$ & $8.06 \pm 0.02$ & $7.44 \pm 0.05$ & 10.994 & $0.000^{* *}$ \\
$\mathrm{~T}\left({ }^{\circ} \mathrm{C}\right)$ & $6.70 \pm 0.82$ & $11.08 \pm 0.27$ & $10.58 \pm 0.08$ & $11.39 \pm 0.13$ & $9.36 \pm 0.22$ & 17.394 & $0.000^{* *}$ \\
$\mathrm{TN}(\mathrm{mg} / \mathrm{L})$ & $0.97 \pm 0.06$ & $1.11 \pm 0.06$ & $0.77 \pm 0.04$ & $0.89 \pm 0.03$ & $0.75 \pm 0.03$ & 2.835 & $0.037^{*}$ \\
$\mathrm{TP}(\mathrm{mg} / \mathrm{L})$ & $0.43 \pm 0.11$ & $0.53 \pm 0.05$ & $0.51 \pm 0.06$ & $0.46 \pm 0.09$ & $0.17 \pm 0.03$ & 5.104 & $0.002^{* *}$ \\
$\mathrm{NH}_{4}{ }^{+}-\mathrm{N}(\mathrm{mg} / \mathrm{L})$ & $0.18 \pm 0.02$ & $0.18 \pm 0.03$ & $0.16 \pm 0.03$ & $0.14 \pm 0.03$ & $0.19 \pm 0.01$ & 1.807 & 0.147 \\
$\mathrm{NO}_{3}{ }^{-}-\mathrm{N}(\mathrm{mg} / \mathrm{L})$ & $0.47 \pm 0.07$ & $0.47 \pm 0.12$ & $0.43 \pm 0.20$ & $0.33 \pm 0.12$ & $0.43 \pm 0.09$ & 0.382 & 0.820 \\
$\mathrm{COD}_{\mathrm{Mn}}(\mathrm{mg} / \mathrm{L})$ & $4.07 \pm 0.04$ & $4.35 \pm 0.08$ & $4.28 \pm 0.05$ & $4.23 \pm 0.06$ & $4.39 \pm 0.04$ & 19.060 & $0.000^{* *}$ \\
$\mathrm{Fe}^{3+}(\mathrm{mg} / \mathrm{L})$ & $0.42 \pm 0.04$ & $0.39 \pm 0.02$ & $0.41 \pm 0.01$ & $0.41 \pm 0.01$ & $0.41 \pm 0.01$ & 3.696 & $0.012^{*}$ \\
$\mathrm{Cu}^{2+}(\mathrm{mg} / \mathrm{L})$ & $0.32 \pm 0.11$ & $0.07 \pm 0.03$ & $0.03 \pm 0.01$ & $0.04 \pm 0.01$ & $0.09 \pm 0.03$ & 4.677 & $0.003^{* *}$ \\
\hline
\end{tabular}

Water transparency (SD), depth (D), electrical conductivity (EC), dissolved oxygen (DO), $\mathrm{pH}$, water temperature (T), total nitrogen (TN), total phosphorus (TP), ammonium nitrogen $\left(\mathrm{NH}_{4}{ }^{+}-\mathrm{N}\right)$, nitrate nitrogen $\left(\mathrm{NO}_{3}^{-}-\mathrm{N}\right)$, chemical oxygen demand $\left(\mathrm{COD}_{\mathrm{Mn}}\right)$ and dissolved iron $\left(\mathrm{Fe}^{3+}\right)$ and dissolved copper $\left(\mathrm{Cu}^{2+}\right), F$ and $p$ values from One-way ANOVA tested by post-hoc test using Tukey HSD ANOVA. ${ }^{*} p<0.05,{ }^{* *} p<0.01$ 
Table 3. The values (mean \pm SE) of environmental factors among sampling seasons in May (spring), July (summer) and September (autumn) in 2015

\begin{tabular}{c|c|c|c|c|c}
\hline & May (Spring) & July (Summer) & September (Autumn) & $\boldsymbol{F}$ & $\boldsymbol{p}$ \\
\hline $\mathrm{SD}(\mathrm{m})$ & $0.69 \pm 0.06$ & $0.96 \pm 0.1$ & $1.06 \pm 0.09$ & 4.974 & $0.012^{* *}$ \\
$\mathrm{D}(\mathrm{m})$ & $8.07 \pm 1.97$ & $10.77 \pm 2.57$ & $10.18 \pm 2.28$ & 0.387 & 0.681 \\
$\mathrm{EC}(\mathrm{mS} / \mathrm{cm})$ & $0.08 \pm 0.02$ & $0.14 \pm 0.02$ & $0.14 \pm 0.02$ & 0.369 & 0.694 \\
$\mathrm{DO}(\mathrm{mg} / \mathrm{L})$ & $7.71 \pm 0.18$ & $7.65 \pm 0.32$ & $8.72 \pm 0.12$ & 7.301 & $0.002^{* *}$ \\
$\mathrm{pH}$ & $7.41 \pm 0.05$ & $7.24 \pm 0.09$ & $7.71 \pm 0.06$ & 11.932 & $0.000^{* *}$ \\
$\mathrm{~T}\left({ }^{\circ} \mathrm{C}\right)$ & $17.47 \pm 0.59$ & $23.35 \pm 0.3$ & $9.82 \pm 0.48$ & 204.801 & $0.000^{* *}$ \\
$\mathrm{TN}(\mathrm{mg} / \mathrm{L})$ & $1.09 \pm 0.1$ & $0.94 \pm 0.07$ & $0.9 \pm 0.04$ & 1.921 & 0.159 \\
$\mathrm{TP}(\mathrm{mg} / \mathrm{L})$ & $0.78 \pm 0.09$ & $0.58 \pm 0.03$ & $0.42 \pm 0.04$ & 8.768 & $0.001^{* *}$ \\
$\mathrm{NH}_{4}{ }^{*}-\mathrm{N}(\mathrm{mg} / \mathrm{L})$ & $0.21 \pm 0.02$ & $0.3 \pm 0.03$ & $0.17 \pm 0.01$ & 10.984 & $0.000^{* *}$ \\
$\mathrm{NO}_{3}{ }^{-}-\mathrm{N}(\mathrm{mg} / \mathrm{L})$ & $0.46 \pm 0.05$ & $0.45 \pm 0.05$ & $0.43 \pm 0.05$ & 0.118 & 0.889 \\
$\mathrm{COD}_{\mathrm{Mn}}(\mathrm{mg} / \mathrm{L})$ & $4.48 \pm 0.04$ & $3.86 \pm 0.09$ & $4.26 \pm 0.04$ & 25.529 & $0.000^{* *}$ \\
$\mathrm{Fe}^{3+}(\mathrm{mg} / \mathrm{L})$ & $0.35 \pm 0.02$ & $0.25 \pm 0.04$ & $0.4 \pm 0.01$ & 8.125 & $0.001^{* *}$ \\
$\mathrm{Cu}^{2+}(\mathrm{mg} / \mathrm{L})$ & $0.23 \pm 0.03$ & $0.17 \pm 0.02$ & $0.11 \pm 0.04$ & 4.556 & $0.016^{*}$ \\
\hline
\end{tabular}

Water transparency (SD), depth (D), electrical conductivity (EC), dissolved oxygen (DO), pH, water temperature $(\mathrm{T})$, total nitrogen $(\mathrm{TN})$, total phosphorus (TP), ammonium nitrogen $\left(\mathrm{NH}_{4}{ }^{+}-\mathrm{N}\right)$, nitrate nitrogen $\left(\mathrm{NO}_{3}{ }^{-}-\mathrm{N}\right)$, chemical oxygen demand $\left(\mathrm{COD}_{\mathrm{Mn}}\right)$ and dissolved iron $\left(\mathrm{Fe}^{3+}\right)$ and dissolved copper $\left(\mathrm{Cu}^{2+}\right), F$ and $p$ values from One-way ANOVA tested by post-hoc test using Tukey HSD ANOVA. ${ }^{*} p<0.05,{ }^{* *} p<0.01$

\section{Macroinvertebrate data characteristics}

During three sampling seasons, we totally collected 904 individuals and identified 11 orders, 23 family and 55 genera or species of macroinvertebrate belong to six FFGs from Tuanjie Reservoir (Table 4). Our findings agreed with the studies of Liu et al. (2019) and Shabani et al. (2019) who also observed the same six FFGs in the same province. According to Rosser and Pearson (2018), aquatic insects were the most abundant group in freshwater ecosystem, Chironomidae especially. Thus, we found that the abundance of Chironomus kiiensisTokunaga (11.62\%) which was absolutely dominant species of macroinvertebrate community structure in Tuanjie Reservoir. The disturbance of Tubificid worms can significantly improve the nutrient level of the water body. These nutrients times of phytoplankton can promote the growth of phytoplankton (Jin et al., 2017), and phytoplankton is the opening bait of benthos. The dependence of benthos and phytoplankton cannot be separated from the change of nutrient concentration (Li et al., 2001; Cortes et al., 2010; Wang et al., 2019).

The highest macroinvertebrate abundance observed in summer at all sampling sites except S5 (in spring), while the lowest abundance presented in autumn except S1 (in spring) (Fig. 2). The temporal changes in macroinvertebrates functional feeding groups (FFGs) of three sampling seasons were shown in Table 5. We found that all macroinvertebrates FFGs top abundance presented in July (summer). Only group GC and total macroinvertebrates abundance showed extremely significant differences $(P<0.01)$, and group FC presented significant differences $(P<0.05)$ during sampling seasons. Both top abundance of group PR and $\mathrm{SH}$ showed at S4, group OM and FC observed at S2. Meanwhile, the top group GC abundance presented at S5, while group SC appeared at S1 (Fig. 3). In three seasons (spring, summer and autumn), the top abundance presented at S5, S2 and S1, respectively (Fig. 4). Both among sampling sites and seasons, FFGs of group GC accounted for most of the proportion (Fig. 5). Based on 
the study of Zhang et al. (2012), the sediment of Danjiangkou reservoir is rich in organic matter, which is one of the important food sources for warworm and chironomid larvae ( $\mathrm{Li}$ et al., 2018). We also found the similar results that relative abundance of group GC (including Tubificidae sp. and Chironomidae sp.) was higher than other groups (Fig. 5) (Benke, 1998; Chaloner et al., 2002).

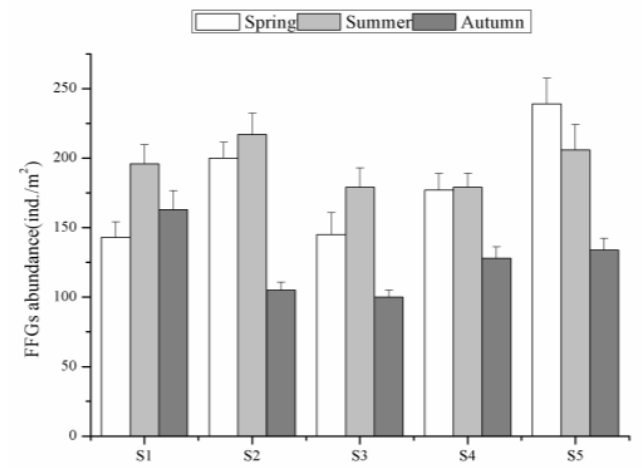

Figure 2. Seasonal variation of macroinvertebrate abundance $\left(\right.$ ind. $\left./ m^{2}\right)$ among sampling sites, error bars meaning standard error
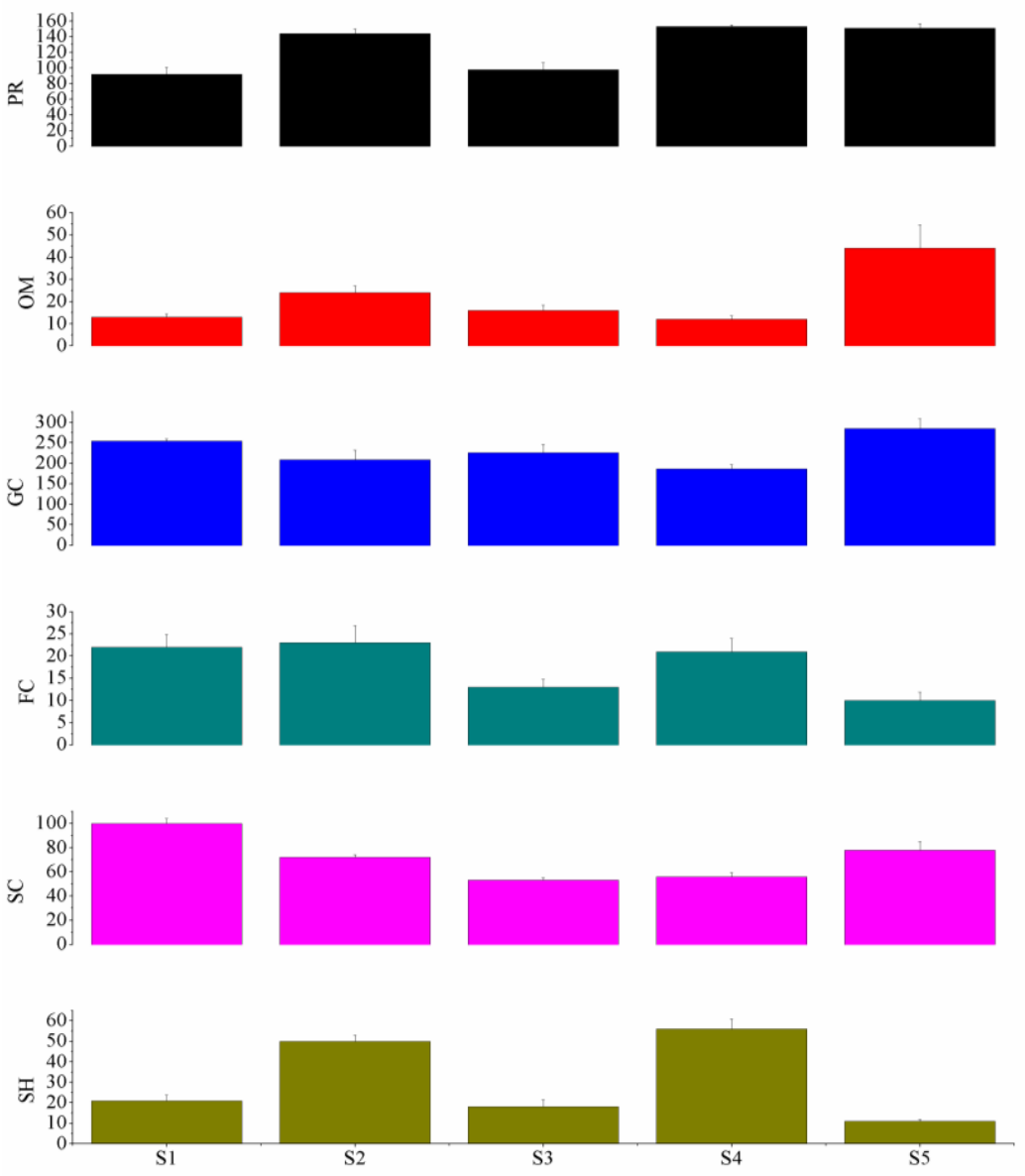

Figure 3. Macroinvertebrate abundance (ind. $\left./ \mathrm{m}^{2}\right)$ among sampling sites. Predators $(P R)$, omnivores (OM), gatherers/collectors (GC), filterers/collectors (FC), scrapers $(S C)$ and shredders $(\mathrm{SH})$ 
Table 4. Macroinvertebrate community structure and abundance ratio in Tuanjie Reservoir in May (spring), July (summer) and September (autumn) in 2015

\begin{tabular}{|c|c|c|c|c|}
\hline Order & Family & Genera or species & FFGs & Ratio \\
\hline \multirow{21}{*}{$\begin{array}{c}\text { Hemiptera } \\
\text { Diptera }\end{array}$} & Corixidae & Corixa substriata & PR & $2.65 \%$ \\
\hline & Chironomidae & Diplocladius sp. & $\mathrm{GC}$ & $2.21 \%$ \\
\hline & & Synorthocladius semivirens & GC & $1.66 \%$ \\
\hline & & Thienemannia gracilis kiffer & GC & $3.43 \%$ \\
\hline & & Chironomus kiiensisTokunaga & GC & $11.62 \%$ \\
\hline & & Chironomus flaviplumus & GC & $7.96 \%$ \\
\hline & & Dicrotendipes pelochloris & GC & $2.10 \%$ \\
\hline & & Chironomus plumosus & OM & $1.33 \%$ \\
\hline & & Cryptochironomus maculipennis & PR & $4.42 \%$ \\
\hline & & Parachironomus arcnatus & PR & $2.43 \%$ \\
\hline & & Eukiefferiella fittkaui & $\mathrm{GC}$ & $1.44 \%$ \\
\hline & & Polypedilum laetum & SH & $1.55 \%$ \\
\hline & & Apsectrotanypus sp. & PR & $2.54 \%$ \\
\hline & & Stictochironomus akizukii & OM & $5.09 \%$ \\
\hline & & Stictochironomus maculipennis & $\mathrm{OM}$ & $0.33 \%$ \\
\hline & & Paracladopelma undine & GC & $1.77 \%$ \\
\hline & & Paracladopelma nigritula & GC & $0.88 \%$ \\
\hline & & Chironomus anthracinus & $\mathrm{GC}$ & $0.66 \%$ \\
\hline & & Micropsectra chuzeprima & GC & $0.66 \%$ \\
\hline & & Tanytarsus chinyensis & FC & $0.44 \%$ \\
\hline & & Tanytarsus signatus & $\mathrm{FC}$ & $1.22 \%$ \\
\hline \multirow[t]{7}{*}{ Trichoptera } & Hydropsychidae & Hydropsyche sp. & FC & $0.66 \%$ \\
\hline & Goeridae & Goera ramosa & $\mathrm{SC}$ & $0.33 \%$ \\
\hline & & Goera kyotonis & $\mathrm{SC}$ & $0.22 \%$ \\
\hline & Rhyacophilidae & Rhyacophila sp. & PR & $0.44 \%$ \\
\hline & Limnephilidae & Apatania sp. & $\mathrm{SC}$ & $1.44 \%$ \\
\hline & & Glyphotaelius admorsus & $\mathrm{SH}$ & $2.54 \%$ \\
\hline & & Stenophylax koizumii & $\mathrm{SH}$ & $0.66 \%$ \\
\hline \multirow[t]{6}{*}{ Ephemeroptera } & Ephemeridae & Ephemera shengmi & $\mathrm{GC}$ & $0.33 \%$ \\
\hline & & Ephemera nigroptera & $\mathrm{GC}$ & $1.11 \%$ \\
\hline & Heptageniidae & Heptagenia sp. & SC & $0.22 \%$ \\
\hline & Baetidae & Baetis sp. & $\mathrm{GC}$ & $1.66 \%$ \\
\hline & Ephemerelliae & Ephemerella nigra & GC & $0.22 \%$ \\
\hline & & Ephemerella fusongensis & GC & $1.11 \%$ \\
\hline Plecoptera & Pelidae & Cyamia sp. & PR & $0.22 \%$ \\
\hline \multirow{3}{*}{ Odonata } & Libellulidae & Epiophcetia superstes & PR & $0.77 \%$ \\
\hline & Libellulidae & Hydrobasileus sp. & PR & $0.44 \%$ \\
\hline & Gomphidae & Anisogomphus sp. & PR & $0.33 \%$ \\
\hline \multirow[t]{2}{*}{ Coleoptera } & Dytiscidae & Cybister japonicus & PR & $1.66 \%$ \\
\hline & Hydrophilidae & Hydrophilus acuminatus & PR & $0.22 \%$ \\
\hline \multirow{6}{*}{$\begin{array}{l}\text { Rhynchobdellida } \\
\text { Tubificida }\end{array}$} & Glossiphoniidae & Helobdella nuda & PR & $2.54 \%$ \\
\hline & Tubificinae & Limnodrilus hoffmeisteri & $\mathrm{GC}$ & $6.75 \%$ \\
\hline & & Limnodrilus claparedeianus & GC & $3.76 \%$ \\
\hline & & Branchiura sowerbyi & $\mathrm{GC}$ & $0.33 \%$ \\
\hline & Naididae & Nais variabilis & GC & $4.42 \%$ \\
\hline & & Dero sp. & GC & $1.00 \%$ \\
\hline \multirow[t]{2}{*}{ Mesogastropoda } & Viviparidae & Bellamya purrificata & SC & $1.88 \%$ \\
\hline & Hydrobiidae & Parafossarulus striatus & $\mathrm{SC}$ & $0.88 \%$ \\
\hline \multirow[t]{7}{*}{ Basommatophora } & Lymnaeidae & Radix auricularia & $\mathrm{SC}$ & $1.00 \%$ \\
\hline & & Radix plicatula & SC & $0.77 \%$ \\
\hline & & Radix swinhoei & SC & $1.44 \%$ \\
\hline & & Radix ovata & $\mathrm{SC}$ & $1.00 \%$ \\
\hline & & Radix lagotis & $\mathrm{SC}$ & $0.88 \%$ \\
\hline & & Galba pervia & $\mathrm{SC}$ & $1.44 \%$ \\
\hline & Planoribidae & Polypylis hemisphaerula & SC & $0.88 \%$ \\
\hline
\end{tabular}

Predators (PR), omnivores (OM), gatherers/collectors (GC), filterers/collectors (FC), scrapers (SC) and shredders (SH) 
Table 5. The abundance (ind. $/ \mathrm{m}^{2}$ ) values (mean $\pm S E$ ) of macroinvertebrate FFGs among sampling seasons in May (spring), July (summer) and September (autumn) in 2015

\begin{tabular}{c|c|c|c|c|c}
\hline & Spring & Summer & Autumn & $\boldsymbol{F}$ & $\boldsymbol{p}$ \\
\hline PR & $41.4 \pm 9.67$ & $49.60 \pm 1.29$ & $36.60 \pm 3.82$ & 1.548 & 0.225 \\
OM & $12.20 \pm 5.85$ & $4.40 \pm 1.91$ & $5.20 \pm 1.83$ & 0.768 & 0.470 \\
GC & $89.4 \pm 9.04 \mathrm{a}$ & $94.00 \pm 7.65 \mathrm{a}$ & $48.60 \pm 11.34 \mathrm{~b}$ & 8.421 & $0.001^{* *}$ \\
FC & $6.40 \pm 1.86$ & $8.60 \pm 2.16$ & $2.80 \pm 1.24$ & 3.460 & $0.041^{*}$ \\
SC & $22.40 \pm 4.60$ & $27.40 \pm 4.45$ & $22.00 \pm 2.53$ & 1.090 & 0.346 \\
SH & $9.00 \pm 4.92$ & $11.40 \pm 3.06$ & $10.80 \pm 3.06$ & 0.223 & 0.801 \\
Total & $180.80 \pm 18.00 \mathrm{a}$ & $195.40 \pm 7.47 \mathrm{a}$ & $126.00 \pm 11.30 \mathrm{~b}$ & 6.633 & $0.003^{* *}$ \\
\hline
\end{tabular}

Predators (PR), omnivores (OM), gatherers/collectors (GC), filterers/collectors (FC), scrapers (SC) and shredders $(\mathrm{SH}), F$ and $p$ values from One-way ANOVA, a and $\mathrm{b}$ mean differences between the seasons were tested by post-hoc test using Tukey HSD ANOVA. ${ }^{*} p<0.05,{ }^{* *} p<0.01$
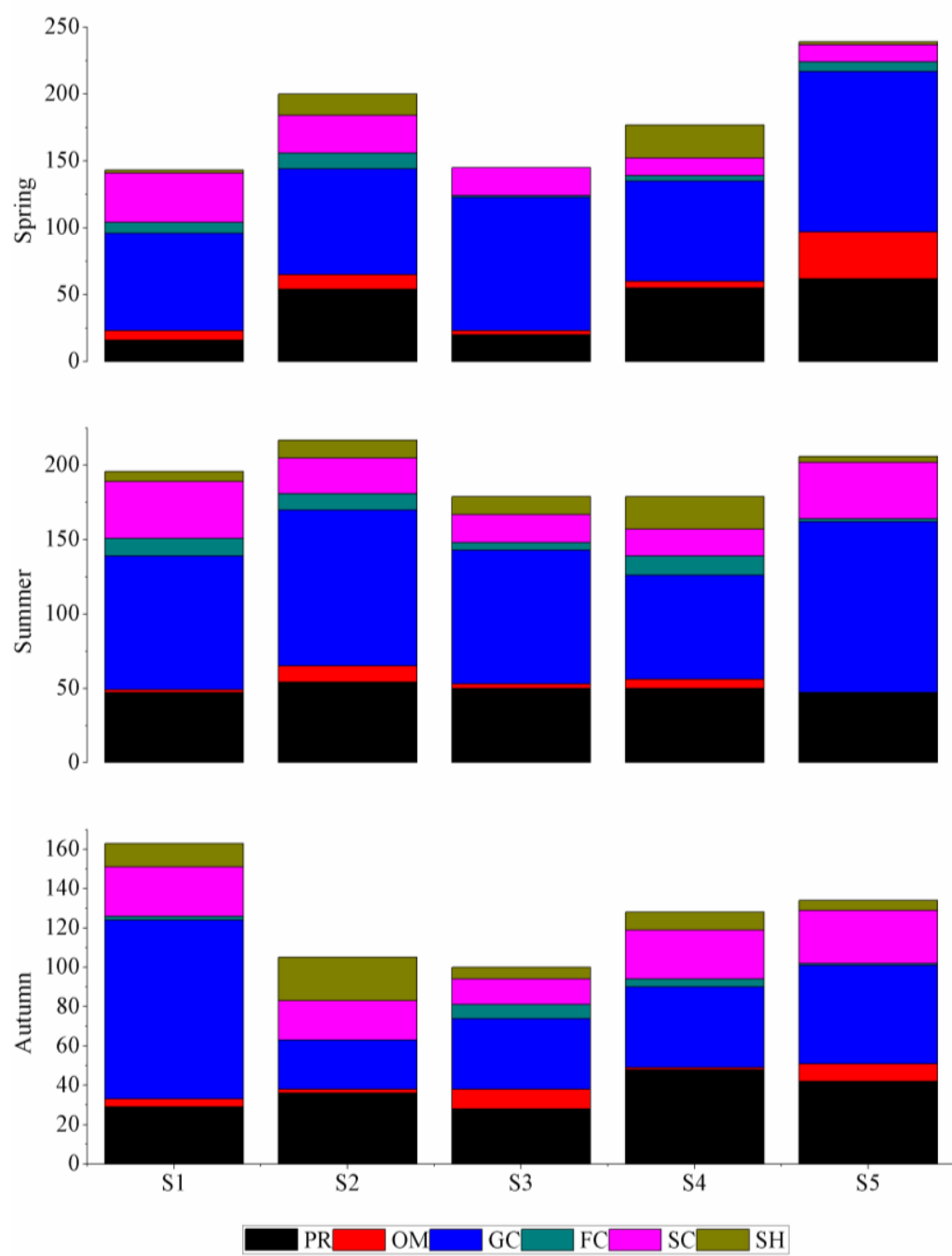

Figure 4. Macroinvertebrate FFGs abundance (ind. $\left./ \mathrm{m}^{2}\right)$ of spring, summer and autumn among sampling sites. Predators (PR), omnivores $(O M)$, gatherers/collectors $(G C)$, filterers/collectors $(F C)$, scrapers ( $S C)$ and shredders $(\mathrm{SH})$ 


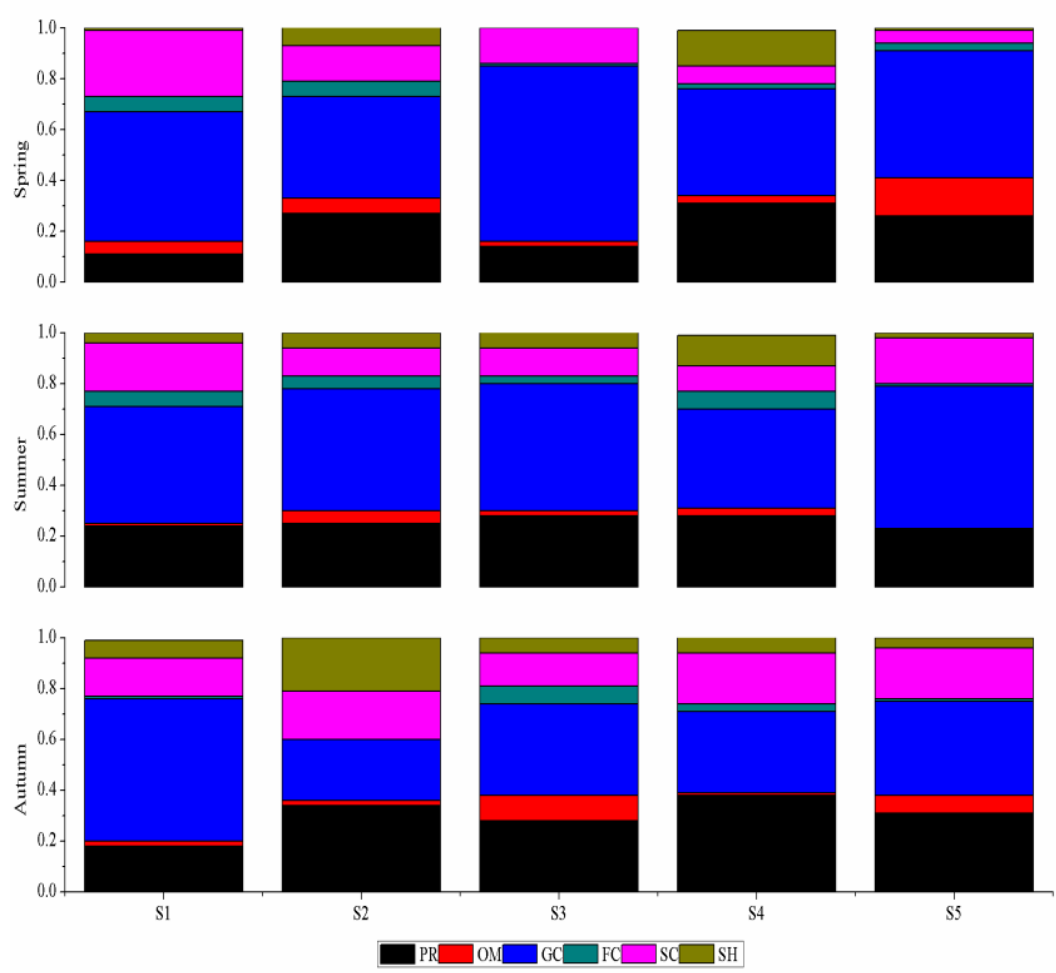

Figure 5. Macroinvertebrate FFGs relative abundance of spring, summer and autumn among sampling sites. Predators (PR), omnivores (OM), gatherers/collectors $(G C)$, filterers/collectors $(F C)$, scrapers $(S C)$ and shredders $(\mathrm{SH})$

\section{Correlation analysis between macroinvertebrate FFGs abundance and environmental factors}

Correlation coefficient is significant if the correlation coefficient is low it did not suppose a close correlation between the studied variables (Pries et al., 2000; Sponseller et al., 2001; Richards et al., 2003; Barquín and Death, 2006). As shown in Table 6, group FC and SC were extremely positive correlated with $\mathrm{T}(\mathrm{r}=0.398)$ and $\mathrm{NO}_{3}{ }^{-} \mathrm{N}$ $(\mathrm{r}=0.381)$. Meanwhile, group $\mathrm{PR}$ and $\mathrm{GC}$ were both positively correlated with $\mathrm{T}$ $(\mathrm{r}=0.372, \mathrm{r}=0.377)$, and group FC was both positively correlated with $\mathrm{TN}(\mathrm{r}=0.355)$ and $\mathrm{NH}_{4}{ }^{+}-\mathrm{N}(\mathrm{r}=0.305)$. By contrast, group $\mathrm{GC}$ was both negatively correlated with DO ( $\mathrm{r}=-$ $0.315)$ and $\mathrm{pH}(\mathrm{r}=-0.299)$.

Li et al. (2018) compared with the results of other reservoirs, it can be seen that the emergence of pollution resistant species, such as Limnodrilus hoffmeisteri and Limnodrilus claparedianus, can play an indicative role in reservoir water quality. With the increase of temperature, the release rate of $\mathrm{NH}_{4}{ }^{+}-\mathrm{N}$ from the Limnodrilus hoffmeisteri (group GC) increased gradually (Gong et al., 2017). Meanwhile, the Limnodrilus hoffmeisteri has strong pollution resistance, can grow and reproduce normally in the low oxygen environment, and even survive for a short time in the low oxygen environment. It is often used as a symbol of organic pollution or eutrophication (Lee, 1970; Wildung et al., 1993; Batzer et al., 2004; Bonada et al., 2007). 
Table 6. Pearson correlation analysis of between FFGs and environmental factors in May (spring), July (summer) and September (autumn) in 2015

\begin{tabular}{|c|c|c|c|c|c|c|c|c|c|c|c|c|}
\hline & SD & D & DO & pH & $\mathbf{T}$ & TN & TP & $\mathrm{NH}_{4}{ }^{+}-\mathrm{N}$ & $\mathrm{NO}_{3}-\mathrm{N}$ & $\operatorname{COD}_{M n}$ & $\mathrm{Fe}^{3+}$ & $\mathrm{Cu}^{2+}$ \\
\hline PR & 0.093 & 0.169 & 0.029 & -0.262 & $0.372^{*}$ & 0.003 & 0.026 & 0.079 & -0.175 & -0.024 & -0.036 & -0.165 \\
\hline $\mathrm{OM}$ & -0.008 & 0.097 & 0.196 & -0.269 & 0.019 & 0.198 & -0.096 & -0.022 & -0.042 & 0.185 & 0.171 & $\mid-0.224$ \\
\hline GC & -0.104 & 0.150 & $-0.315^{*}$ & $-0.299^{*}$ & $0.377^{*}$ & 0.263 & 0.166 & 0.217 & -0.051 & 0.000 & -0.140 & $\mid-0.009$ \\
\hline FC & -0.263 & -0.131 & -0.201 & & $0.398^{* * *}$ & $0.355^{*}$ & 0.047 & $0.305^{*}$ & -0.015 & 0.11 & -0.1 & 0.001 \\
\hline SC & 0.189 & 0.090 & -0.229 & -0.060 & 0.084 & -0.055 & 0.095 & 0.223 & $0.381^{* * *}$ & -0.263 & -0.122 & 0.035 \\
\hline SH & 0.151 & 0.073 & -0.185 & 0.138 & -0.007 & -0.224 & 0.037 & -0.151 & 0.069 & -0.133 & -0.272 & 0.015 \\
\hline
\end{tabular}

Water transparency (SD), depth (D), electrical conductivity (EC), dissolved oxygen (DO), $\mathrm{pH}$, water temperature $(\mathrm{T})$, total nitrogen $(\mathrm{TN})$, total phosphorus $(\mathrm{TP})$, ammonium nitrogen $\left(\mathrm{NH}_{4}{ }^{+}-\mathrm{N}\right)$, nitrate nitrogen $\left(\mathrm{NO}_{3}-\mathrm{N}\right)$, chemical oxygen demand $\left(\mathrm{COD}_{\mathrm{Mn}}\right)$ and dissolved iron $\left(\mathrm{Fe}^{3+}\right)$ and dissolved copper $\left(\mathrm{Cu}^{2+}\right)$, predators (PR), omnivores (OM), gatherers/collectors (GC), filterers/collectors (FC), scrapers (SC) and shredders (SH). ${ }^{*} \mathrm{p}<0.05,{ }^{* *} \mathrm{p}<0.01$

There are many factors affecting the community structure, density, biomass and biodiversity of benthos (Shen et al., 2015; Rodrigues et al., 2019; Oremo et al., 2019; Ruaro et al., 2019; Santonja et al., 2020; Taylor et al., 2020). The summary of Monte Carlo test showed that the first canonical axis and all canonical axes were significant ( $F=5.059, p=0.002 ; F=1.260, p=0.002 ; 499$ random permutations). The eigenvalues of the four axes were $0.140,0.095,0.055,0.032$, respectively (Table 7 ). The speciesenvironment correlations for Axis 1 and Axis 2 were 0.674 and 0.753 , respectively. The first two axes account for $23.6 \%$ of FFGs data relation (axis 1: 14.0\%, axis 2: 9.6\%) and $68.2 \%$ of FFGs-environment data (axis 1: $40.6 \%$, axis 2: 27.6\%). The Axis1 was mainly positively correlated with $\mathrm{T}, \mathrm{NH}_{4}{ }^{+}-\mathrm{N}$ and $\mathrm{TN}$, and negatively correlated with $\mathrm{pH}, \mathrm{SD}$ and D. TN content reflects the nutritional status of water body. A large number of studies showing that the input of nutrients leads to a higher TN, and the nutritional status of water body is closely related to the secondary productivity of benthos (Heino, 2005; Benke, 2010; Dolbeth et al., 2012; Hughes et al., 2012). The Axis 2 was positively correlated with $\mathrm{T}$ and $\mathrm{NH}_{4}{ }^{+}-\mathrm{N}$, and negatively correlated with $\mathrm{Fe}^{3+}$, DO and COD ${ }_{M n}$. Groups FC was mainly influenced by $\mathrm{T}$ and $\mathrm{NH}_{4}{ }^{+}-\mathrm{N}$, and group $\mathrm{GC}$ was influenced by EC. While group PR was mainly influenced by $\mathrm{TN}$, and $\mathrm{OM}$ was influenced by $\mathrm{COD}_{\mathrm{Mn}}$. The main environmental factors were $\mathrm{T}, \mathrm{NH}_{4}{ }^{+}-\mathrm{N}, \mathrm{Fe}^{3+}, \mathrm{DO}$ and $\mathrm{COD}_{\mathrm{Mn}}$ in Tuanjie Reservoir (Fig. 6).

Table 7. RDA results of macroinvertebrate FFGs abundance in May (spring), July (summer) and September (autumn) in 2015

\begin{tabular}{c|c|c|c|c}
\hline Axes & $\mathbf{1}$ & $\mathbf{2}$ & $\mathbf{3}$ & $\mathbf{4}$ \\
\hline Eigenvalues & 0.140 & 0.095 & 0.055 & 0.032 \\
Species-environment correlations & 0.674 & 0.753 & 0.716 & 0.366 \\
$\begin{array}{c}\text { Cumulative percentage variance } \\
\text { of species data }\end{array}$ & 14.0 & 23.6 & 29.0 & 32.2 \\
of species-environment relation & 40.6 & 68.2 & 84.0 & 93.2 \\
\hline
\end{tabular}




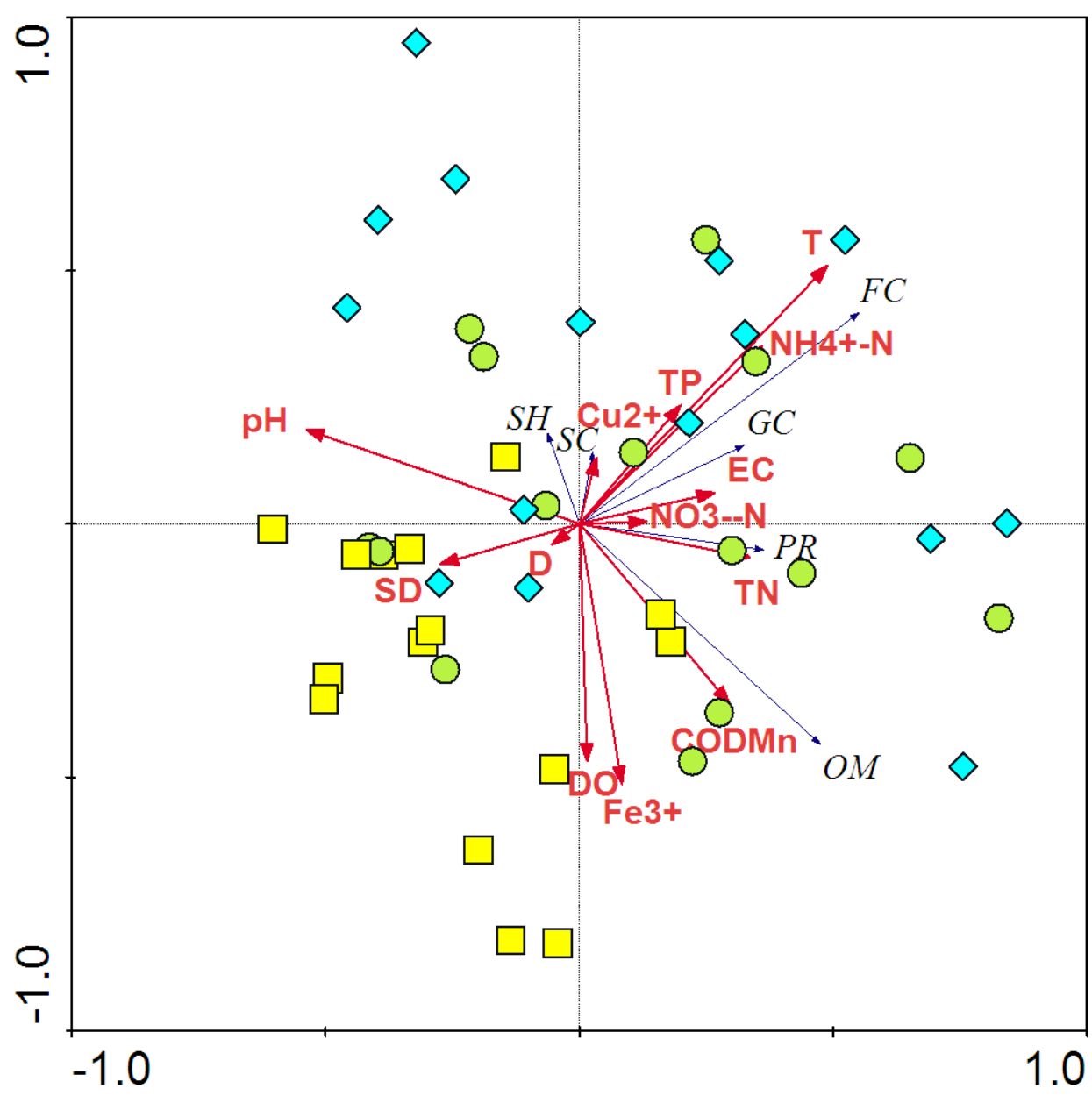

Figure 6. RDA bioplot of macroinvertebrate FFGs and environmental factors. Green circle: Spring; Blue diamond: Summer; Yellow square: Autumn. Water transparency $(S D)$, depth $(D)$, electrical conductivity $(E C)$, dissolved oxygen $(D O), p H$, water temperature $(T)$, total nitrogen $(\mathrm{TN})$, total phosphorus (TP), ammonium nitrogen $\left(\mathrm{NH}_{4}{ }^{+}-\mathrm{N}\right)$, nitrate nitrogen $\left(\mathrm{NO}_{3}{ }^{-} \mathrm{N}\right)$, chemical oxygen demand $\left(C O D_{M n}\right)$ and dissolved iron $\left(\mathrm{Fe}^{3+}\right)$ and dissolved copper $\left(\mathrm{Cu}^{2+}\right)$, predators $(P R)$, omnivores $(O M)$, gatherers/collectors $(G C)$, filterers/collectors $(F C)$, scrapers $(S C)$ and shredders $(\mathrm{SH})$

\section{Conclusion}

During the study period, we totally collected 904 individuals and identified 11 orders, 23 family and 55 genera or species of macroinvertebrate belong to six FFGs (PR, OM, GC, FC, SC and SH) from study area. The mean values of SD, DO, pH, T, $\mathrm{TP}, \mathrm{COD}_{\mathrm{Mn}}$ and $\mathrm{Cu}^{2+}$ were extremely significant difference among sampling sites $(P<0.01)$. The main environmental factors were $\mathrm{T}, \mathrm{NH}_{4}{ }^{+}-\mathrm{N}, \mathrm{Fe}^{3+}, \mathrm{DO}$ and $\mathrm{COD}_{\mathrm{Mn}}$ in Tuanjie Reservoir. Our findings can provide strong recommendations for scientific basis for the biological resources protection and water quality operation and management of Tuanjie reservoir for future.

Acknowledgements. We sincerely acknowledge the National Key Research and Development Program of China (2016YFC0500406). 


\section{REFERENCES}

[1] An, R., Wang, F. Y., Yu, H. X., Ma, C. X. (2016): Characteristics and physical factors of phytoplankton functional groups in Small Xingkai Lake. - Res. Environ. Sci. 29(7): 985994. (in Chinese with English abstract).

[2] An, R., Wang, F. Y., Yu, H. X., Ma, C. X. (2017): Seasonal dynamics of zooplankton functional groups and their relationships with environmental factors in the sanhuanpao wetland reserve. - Acta Ecologica Sinica 37(6): 1851-1860. (in Chinese with English abstract).

[3] Barquín, J., Death, R. G. (2006): Spatial patterns of macroinvertebrate diversity in new zealand springbrooks and rhithral streams. - J. of the Nor. Ameri. Benth. Soci. 25(4): 768-786.

[4] Batzer, D. P., Palik, B. J., Buech, R. (2004): Relationships between environmental characteristics and macroinvertebrate communities in seasonal woodland ponds of minnesota. - J. of the Nor. Ameri. Benth. Soci. 23(1): 50-68.

[5] Benke, A. C. (1998): Production dynamics of riverine chironomids: Extremely high biomass turnover rates of primary consumers. - Ecology 79(3): 899-910.

[6] Benke, A. C. (2010): Secondary production as part of bioenergetic theory-contributions from freshwater benthic science. - River Res. Appl. 26: 36-44.

[7] Bonada, N., Rieradevall, M., Prat, N. (2007): Macroinvertebrate community structure and biological traits related to flow permanence in a mediterranean river network. Hydrobiologia 589: 91-106.

[8] Buss, D. F., Baptista, D. F., Silveira, M. P., Nessimian, J. L., Dorvillé, L. F. M. (2002): Influence of water chemistry and environmental degradation on macroinvertebrate assemblages in a river basin in south-east Brazil. - Hydrobiologia 481: 125-136.

[9] Chaloner, D., Wipfli, M. S., Caouette, J. P. (2002): Mass loss and macroinvertebrate colonization of pacific salmon carcasses in south-eastern Alaska streams. - Fresh. Bio. 47(2): 263-273.

[10] Chen, J. X., Sun, X., Chai, Q. Y., Yu, H. X., Chai, F. Y., Yu, T., Chen, J. G. (2019): Phytoplankton community structure and water quality assessment in Tuanjie Reservoir. J. Northeast Fore. Univ. 47(03): 85-88. (in Chinese with English abstract).

[11] Chi, S. Y., Peng, J. H., Wan, C. Y., Zou, X., Li, M. (2009): Preliminary study on macrozoobenthos of Sandaohe Reservoir, Hubei Province. - J. Lake Sci. 21(5): 705-712. (in Chinese with English abstract).

[12] Cortes, R. M. V., Ferreira, M. T., Oliveira, S. V., Oliveira, D. (2010): Macroinvertebrate community structure in a regulated river segment with different flow conditions. - River Res. App. 18(4): 367-382.

[13] Cummins, K. W. (1974): Structure and function of stream ecosystems. - Bio Science 24: 631-641.

[14] Dolbeth, M., Cusson, M., Sousa, R. (2012): Secondary production as a tool for better understanding of aquatic ecosystems. - Canad. J. of Fish. Aqu. Sci. 69: 1230-1253.

[15] Duan, X. H., Wang, Z. Y., Xu, M. Z. (2010): Benthic macroinvertebrate and application in the assessment of stream ecology. - Tsinghua University Press, Beijing. (in Chinese).

[16] Duran, M. (2006): Monitoring water quality using benthic macroinvertebrates and physiochemical parameters of Behzat stream in Turkey. - Pol. J. Environ. Stud. 15(5): 709-717.

[17] Epler, J. H. (2001): Identification manual for the larval Chironomidae (Diptera) of North and South Carolina. - FEBS Letters 81(2).

[18] Gong, Z. J., Liu, J. S., Li, Y., Cai, Y. J., Xue, Q. J., Xu, H. (2017): Potential excretion of $\mathrm{NH}_{4}{ }^{+}-\mathrm{N}$ and $\mathrm{PO}_{4}{ }^{3-}-\mathrm{P}$ by Limnodrilus hoffmeisteri Claparède in Lake Taihu. - J. Lake Sci. 29(2): 389-397. (in Chinese with English abstract).

[19] Han, B. P. (2010): Reservoir ecology and limnology in China: A retrospective comment. - J. Lake Sci. 22(2): 151-160. (in Chinese with English abstract). 
[20] Han, B. P., Shi, Q. C., Chen, W. X. (2016): China's Ecology and Water Quality Management of Reservoir. - Beijing: Science Press. (in Chinese).

[21] Heino, J. (2005): Functional biodiversity of macroinvertebrate assemblages along major ecological gradients of boreal headwater streams. - Fresh. Bio. 50(9): 1578-1587.

[22] Hu, T., Wei, K. J., Zhang, G. R., Xu, L., Ma, X. F., Zhao, J. W., Liu, C., Wu, X. H. (2018): Macroinvertebrate communities and bioassessment of water quality in Miyun Reservoir, Beijing. - J. Hydroecol. 39(4): 79-88. (in Chinese with English abstract).

[23] Hughes, R., Herlihy, A., Gerth, W., Pan, Y. D. (2012): Estimating vertebrate, benthic macroinvertebrate, and diatom taxa richness in raftable pacific northwest rivers for bioassessment purposes. - Envir. Moni. Assess. 184(5): 3185-3198.

[24] Ji, L., Song, C., Cao, X., Zhou, Y., Deng, D. (2015): Spatial variation in nutrient excretion by macrozoobenthos in a Chinese large shallow lake (Lake Taihu). - J. Freshw. Ecol. 30(1): 169-180.

[25] Jin, H., Gu, J., Cai, Y. J., He, H., Ning, X. Y., Shen, R. J., Yang, G. J., Li, K. Y. (2017): Inhibiting effect of benthic algae on the bioturbation of Limnodrilus hoffmeisteri. - Acta Sci. Circum. 37(6): 2055-2060. (in Chinese with English abstract).

[26] Lee, G. F. (1970): Factors affecting the transfer of materials between water and sediments. - University of Wisconsin, Water Resources Center, Eutrophication Information Program.

[27] Li, J., Herlihy, A., Gerth, W., Kaufmann, P., Gregory, S., Urquhart, S., Larsen, D. P. (2001): Variability in stream macroinvertebrate at multiple spatial scales. - Fresh. Bio. 46(1): 87-97.

[28] Li, B., Zhang, M., Cai, Q. H. (2018): Spatial distribution of macrobenthic secondary production of Danjiangkou Reservoir. - Asi. J. of Ecot. 13(4): 22-29. (in Chinese with English abstract).

[29] Li, Y. G., Hu, Q. J., Qu, J. Q., Zong, H. M., Wang, J., Zhang, Q. J. (2018): Community structure and spatial-temporal variation of zoobenthos in Miyun Reservoir, Beijing. - J. Hydroecol. 39(5): 32-38. (in Chinese with English abstract).

[30] Liu, Q. G., Zha, Y. T., Chen, L. Q., Gu, Z. M., Jia, Y. Y., Hu, Z. J. (2012): Macrozoobenthos community structure and its indicative significance in water quality bio-assessment of Fenshuijiang Reservoir, Zhejiang Province of East China. - Chin. J. Appl. Ecol. 23(5): 1377-1384. (in Chinese with English abstract).

[31] Liu, M. H., Meng, Y., Cao, J. J., Cui, X. B., Al, M. N. (2019): Functional traits of macroinvertebrates in Naolihe Wetland. - J. Northeast Fore. Univ. 47(01): 76-82. (in Chinese with English abstract).

[32] Mangadze, T., Bere, T., Mwedzi, T. (2016): Choice of biota in stream assessment and monitoring programs in tropical streams: a comparison of diatoms, macroinvertebrates and fish. - Ecol. Ind. 63(4): 128-143.

[33] MEP. (2002): China national environmental quality standards for surface water. GB3838-2002 (in Chinese).

[34] Morse, J. C., Yang, L. F., Tian, L. X. (1994): Aquatic insects of China useful for monitoring water quality. - Nanjing: Hohai University Press.

[35] Oremo, J., Orata, F., Owino, J., Shivoga, W. (2019): Assessment of heavy metals in benthic macroinvertebrates, water and sediments in River Isiukhu, Kenya. - Envir. Moni. Asse. 191(11): 646-658.

[36] Petridis, D., Sinis, A. (1993): Benthic macrofauna of Tavropos Reservoir (central Greece). - Hydrobiologia 262(1): 1-12.

[37] Pires, A. M., Cowx, I. G., Coelho, M. M. (2000): Benthic macroinvertebrate communities of intermittent streams in the middle reaches of the guadiana basin (portugal). Hydrobiologia 435(1): 167-175.

[38] Plafkin, J. L., Barbour, M. T., Porter, K. D. (1989): Rapid bioassessment protocols for use in streams and rivers: benthic macroinvertebrates and fish. - Washington DC: US Environmental Protection Agency. 
[39] Poff, N. L., Olden, J. D., Vieira, N. K. M., Finn, D. S., Simmons, M. P., Kondratieff, B. C. (2006): Functional trait niches of North American lotic insects: traits-based ecological applications in light of phylogenetic relationships. - The North Ameri. Benth. Soci. 25(4): 730-755.

[40] Richards, C., Haro, R., Johnson, L., Host, G. (2003): Catchment and reach-scale properties as indicators of macroinvertebrate species traits. - Fresh. Bio. 37(1): 219-230.

[41] Rodrigues, C., Guimaraes, L., Vieira, N. (2019): Combining biomarker and community approaches using benthic macroinvertebrates can improve the assessment of the ecological status of rivers. - Hydrobiologia 839: 1-24.

[42] Rosser, Z. C., Pearson, R. G. (2018): Hydrology, hydraulics and scale influence macroinvertebrate responses to disturbance in tropical streams. - J. Fresh. Ecol. 33(1): 117.

[43] Ruaro, R., Gubiani, É. A., Cunico, A. M., Higuti, J., Moretto, Y., Piana, P. A. (2019): Unified multimetric index for the evaluation of the biological condition of streams in southern brazil based on fish and macroinvertebrate assemblages. - Envir. Manag. 64(5): 661-673.

[44] Santonja, M., Rodríguez-Pérez, H., Le Bris, N., Piscart, C. (2020): Leaf nutrients and macroinvertebrates control litter mixing effects on decomposition in temperate streams. Ecosystems 23: 400-416.

[45] Shabani, I. E., Liu, M. H., Yu, H. X., Muhigwa, J., Geng, F. F. (2019): Benthic macroinvertebrate diversity and functional feeding groups in relation to physicochemical factors in Sanjiang plain wetlands, northeast China. - Appl. Ecol. Environ. Res. 17(2): 3387-3402.

[46] Shen, H. Y., Cao, Z. H., Liu, J. W., Wang, W. H., Zhang, Y. (2015): Relationship between functional feeding groups of macroinvertebrates and environmental factors in Taizi River basin. - China Envir. Sci. 35(2): 579-590. (in Chinese with English abstract).

[47] Sponseller, R. A., Benfield, E. F., Valett, H. M. (2001): Relationships between land use, spatial scale and stream macroinvertebrate communities. - Fresh Bio. 46(10): 1409-1424.

[48] Sun, X., Chai, F. Y., Mwagona, P. C., Shabani, I. E., Hou, W. J., Li, X. Y., Yu, H. X. (2019): Seasonal variations of zooplankton functional groups and relationship with environmental factors in a eutrophic reservoir from cold region. - Appl. Ecol. Environ. Res. 17(4): 7727-7740.

[49] Taylor, M. D., Fowler, A. M., Suthers, I. (2020): Insights into fish auditory structurefunction relationships from morphological and behavioural ontogeny in a maturing sciaenid. - Mari. Biol. 167(2): 163-176.

[50] Wang, H. B., Huang, X. L., Wu, J. S., Du, X., Wang, Q. S., Song, D., Huo, T. B. (2019): Effect of agricultural non-point source pollution on plankton and zoobenthos communities in a reservoir in Morin Dawa Daur Autonomous Banner. - Chin. J. Fish. 32(4): 55-62. (in Chinese with English abstract).

[51] Wildung, R. E., Schmidt, R. L. (1993): Phosphorus release from lake sediments. - USA Environmental Protection Agency, Office of Research and Monitoring.

[52] Zhang, M., Cai, Q. H., Xu, Y. H. (2012): Zonation and its influencing factors of a large subtropical reservoir (Danjiangkou Reservoir in central China), based on macroinvertebrates. - Fres. Envir. Bull. 22(6): 2095-2104. 